\title{
ОБЗОРЫ
}

\section{Концепция рыночной эффективности: теоретическое и эмпирическое обоснование и роль в создании стоимости}

\author{
Лебедев О.В. ${ }^{28}$
}

В статье приведен обзор развития концепџии эффрективности рынков с позищии эволючии научных взглядов на моделирование динамики иен на финансовые активы, представлен анализ критики конщепщии рыночной эффективности, рассмотрены особенности создания стоимости в условиях низкоэффективных финансовых рынков.

$$
\text { JEL: } G 14, G 30
$$

Ключевые слова: гипотеза рыночной эффрективности, случайное блуждание, мартингал, создание стоимости

Финансовые теории часто базируются на явных или неявных предположениях, касающихся вероятностной структуры случайных процессов, лежащих в основе динамики ценовых данных (доходность акций, курсы валют, процентные ставки и др.). Так, гипотеза рыночной эффективности (efficient market hypothesis EMH) предполагает, что эволюция цен может быть смоделирована как случайное блуждание (Bachelier, 1900; Fama, 1970) или мартингальный процесс (Samuelson, 1965; Mandelbrot, 1966). Более того, теоретические модели, такие как среднедисперсионный подход в рамках портфельной теории, модель ценообразования капитальных активов (САРМ), модель ценообразования опционов Блэка Шоулза основаны на предпосылке о нормальности распределения, некоррелированности и стационарности рыночных цен. В то же время эмпирические исследования обнаружили расхождения с данными предположениями относительно вероятностной структуры. Ценовые данные часто демонстрируют положительный эксцесс (leptokurticity), непостоянство дисперсии, кластеризацию волатильности (volatility clustering) (Kendall, 1953; Mandelbrot, 1963; Pagan, 1996). Элиминировать первые два несоответствия при моделировании цен было предложено через замену нормального распределения на семейство Парето - Леви распределений. Изучение группирования волатильности привело к развитию моделей динамической волатильности (Bollerslev et al., 1992). Обнаруженные расхождения привели к модификациям моделей случайного блуждания при сохранении свойств непредсказуемости изменения цен (прямое следствие ЕМН); их развитие с позиции эволюции научной мысли относительно моделирования цен на финансовые активы (этапизация в терминах (Andreou et al., 2001)) представлено на рисунке 1.

\footnotetext{
${ }^{28}$ Магистрант кафедры финансового менеджмента Казанского государственного финансово-экономического института.
} 


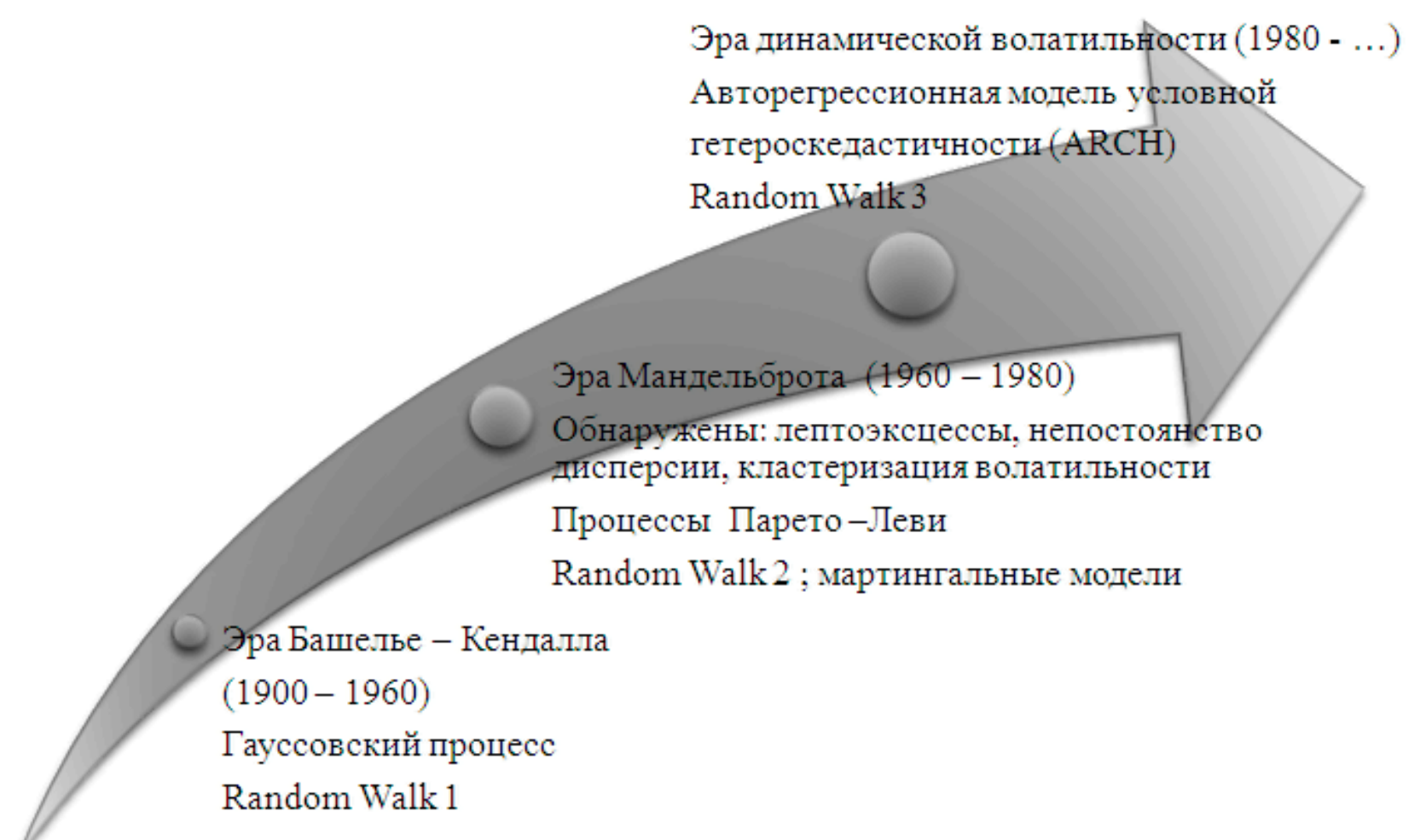

Рисунок 1. Моделирование финансовых рядов и ЕМН

\section{Становление концепции эффективности финансовых рынков}

Истоки гипотезы эффективности рынка обычно относят к диссертации Луи Башелье «Теория спекуляций» (Bachelier, 1900), где он изложил ряд соображений, касающихся случайного колебания цен на акции. Уже тогда он утверждал, что «математическое ожидание спекулянта равно нулю» и что «прошлые, настоящие и ожидаемые будущие события уже отражены в цене».

Строго говоря, Л. Башелье предполагал, что процесс изменения цен $S=\left(S_{t}\right)_{t \geq 0}$ есть случайный процесс. Изучая временной ряд цен $S_{t}^{(\Delta)}, \mathrm{t}=0, \Delta, 2 \Delta$ (с интервалом времени $\left.\Delta\right)$, он отмечает, что $S_{t}^{(\Delta)}-S_{t-\Delta}^{(\Delta)}$ имеют (в статистическом смысле) нулевое среднее и флуктуации $\left|S_{t}^{(\Delta)}-S_{t-\Delta}^{(\Delta)}\right|_{\text {порядка } \sqrt{\Delta}}$.

Таким свойством обладает случайное блуждание:

(1) $S_{t}^{(\Delta)}=S_{0}+\xi_{\Delta}+\xi_{2 \Delta}+\ldots+\xi_{k \Delta}$,

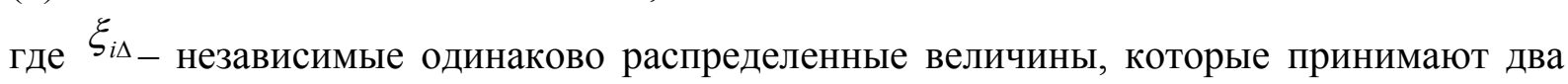
значения $\pm \sigma \sqrt{\Delta}$ с вероятностями $1 / 2$.

Предельный переход по $\Delta \rightarrow 0$ приводит к случайному процессу:

$$
S_{t}=S_{0}+\sigma W_{t}, t \geq 0
$$

где $W=\left(W_{t}\right)_{t \geq 0}$ есть не что иное, как рассмотренное Л. Башелье броуновское движение $^{29}$ (или винеровский процесс - назван в честь Н. Винера, построившего этот

\footnotetext{
${ }^{29}$ В 1827 году английский ботаник Роберт Броун заметил, как зерна пыльцы, растворенные в воде, движутся в энергичной, но случайной манере. Это происходит из-за случайных колебаний в количестве водных молекул, бомбардирующих зерна пыльцы с различных направлений. Если же мы спроецируем это наблюдение на финансовые рынки, то цены бомбардируются мелкими частицами новостей.
} 
процесс и описавшего его математически в 1923 году) (Ширяев, 1998). Работы Л. Башелье намного опередили свое время и были забыты почти на полвека.

Теории броуновского движения в различных модификациях, предполагающие непрерывность временного ряда, широко используются в моделировании в управлении корпоративными финансами (Sawyer, 1993).

В первой половине прошлого века были добыты некоторые эмпирические доказательства случайности блуждания цен. Так, Л. Башелье находит, что колебание цен и на сырьевые товары носит случайный характер, а Воркинг (Working, 1934) и Коулс и Джонс (Cowles and Jones, 1937) доказывают, что такими же характеристиками обладают и колебания цен на акции. Коулс (Cowles, 1933) также проанализировал эффективность прогнозов специалистов по инвестиционному делу и пришел к выводу, что прогнозы на фондовом рынке несостоятельны. Позднее он же доказывает, что специалистам в области инвестиций не «побить» рынок. Воркинг (Working, 1949) подтверждает доводы Коулса о неумении аналитиков делать успешные прогнозы.

Исследователи приводили весьма эксцентричные аналогии колебания курса акции с «повседневными» наблюдениями проявлений случайности. Так, К. Пирсону они напоминали пошатывающегося пьяного, пытающегося ухватиться за фонарный столб (Pearson, 1905). Другие же полагают, что у курса не больше памяти, чем у рулетки (Working, 1934) или пары костей (MacCauley, 1925), и что каждое наблюдение здесь независимо от предыдущего наблюдения. По их мнению, сегодняшнее движение цен не зависит от того, что произошло минуту назад, вчера или позавчера.

А уже в 1953 году М.Дж. Кендалл (Kendall, 1953) проанализировал 22 ценовые серии (используя, в отличие от Л. Башелье, логарифмы цен) с недельным интервалом и обнаружил, что они, по сути, случайны. Он пришел к выводу, что «в рядах цен, которые наблюдаются на достаточно коротком интервале, изменения настолько велики, что не может быть выявлена какая-либо система», и находит такое поведение близким к случайному блужданию. Выражаясь формально, М.Дж. Кендалл утверждал, что логарифмы цен $S=\left(S_{n}\right)$ ведут себя как случайное блуждание; то есть если $h_{n}=\ln \frac{S_{n}}{S_{n-1}}$, то:

$$
S_{n}=S_{0} e^{H n}, n \geq 1,
$$

где $\mathrm{H}_{\mathrm{n}}$ есть сумма независимых случайных величин $\mathrm{h}_{1}, \ldots, \mathrm{h}_{\mathrm{n}}$.

В 1959 году М. Осборн (Osbourne, 1959) независимо от М.Дж. Кендалла приходит, в сущности, к тем же самым выводам, отмечая, что не сами цены, а их логарифмы подчиняются броуновскому движению. Непредсказуемость динамики цен на акции также демонстрировалась в последующих исследованиях (Roberts, 1959; Working, 1960; Cowles, 1960; Moore, 1962).

Однако появлялась и критика теории случайного блуждания цен на акции. В 1962 году А. Мур обнаруживает незначительную отрицательную автокорреляцию доходности отдельных акций, но слабую положительную автокорреляцию доходности рыночного индекса (Moore, 1962). Грейнджер и Моргенштерн (Granger and Morgenstern, 1963) находят, что краткосрочные колебания цен повинуются случайному блужданию, однако долгосрочные - нет. Впрочем, Х. Воркинг (Working, 1960) и Б. Александер (Alexander, 1961) показывали, что использование усреднения «внедряет» автокорреляцию, отсутствующую в реальных временных рядах.

В целях доказательства случайности колебаний цен было предпринято немало попыток описать их и иными типами распределения случайных величин, помимо нормального. Мандельброт находит, что распределение изменений цен на акции слишком «остроконечно», чтобы аппроксимироваться гауссовским процессом. Также он утверждает, что «хвосты» распределения цен на акции являются длинными («тяжелыми») по сравнению с нормальным. Позднее он тестирует новую модель поведения цен. Он использует натуральные логарифмы цен, а также заменяет нормальное распределение устойчивым распределением Парето - Леви 
(Mandelbrot, 1963, 1969).

Впрочем, использование семейства Парето - Леви распределений при моделировании доходностей позже было поставлено под сомнение. Ряд исследований (Blattberg and Gonedes, 1974; Akgiray and Booth, 1988) показал, что свойство инвариантности характеристической экспоненты (определяет «пиковость» плотности вероятности) нарушается. Характеристическая экспонента не остается постоянной при переходе от еженедельной к ежемесячной доходности. Кроме того, было показано, что t-распределение Стьюдента обеспечивает лучшую аппроксимацию доходности акций, по сравнению с распределением Парето - Леви (Blattberg and Gonedes, 1974; Hagerman, 1978; Tucker, 1992).

Б. Мандельброт также, проведя статистический анализ финансовых временных рядов, отмечает, что многие из них обладают свойствами фрактальности (статистического самоподобия), проявляющимися в том, что, образно говоря, их «части устроены так же, как и целое (Mandelbrot, 1963)» ${ }^{30}$.

По мере того как накапливались знания по поведению цен на финансовые активы на конкурентном рынке, модель случайного блуждания постепенно стали рассматривать как совокупность соответствующих эффективности рынка данных. Так, П. Самуэльсон отмечает, «что на конкурентных рынках для каждого продавца есть покупатель, и если бы можно было быть уверенным в росте цен, то они бы уже выросли». Строго говоря, он сделал предположение о мартингальности цен (т.е. лучший прогноз цены на «завтра» есть значение цены «сегодня»). Он представляет формальное доказательство того, что ожидаемые цены колеблются случайным образом, если выдерживается ряд строгих предпосылок: экономические агенты имеют одинаковые и постоянные временные предпочтения, одинаково оценивают ожидаемые доходности и нейтральны к риску (Samuelson, 1965). Схожие доказательства того, что при ряде предпосылок доходность акций будет соответствовать справедливой игре (разности мартингалов) были представлены Б. Мандельбротом (Mandelbrot, 1966), Ю. Фамой (Fama 1965, 1970). Впрочем, более качественное описание фактической динамики цен обеспечивается субмартингальной моделью, в соответствии с которой инвестор ожидает получение нулевой сверхдоходности (доходности с учетом рисковости активов). Отметим, что мартингальные модели использовались и для аппроксимации динамики цен на производные ценные бумаги (Merton, 1990), а внимание к мартингалам в теории финансов привело к развитию тесно связанных с ними моделей - теорий случайного блуждания (рассмотрены подробно ниже).

А уже в 1967 году Г. Робертс предложил термин «гипотеза рыночной эффективности» (EMH). Окончательное же закрепление ЕМН происходит в 1970 году, когда Юджин Фама (Eugene Fama) публикует статью «Efficient Capital Markets: A Review of Theory and Empirical Work». Он определяет эффективный рынок как рынок, на котором цены всегда в полной мере отражают всю доступную информацию, и как рынок, на котором инвесторы не могут получать сверхдодход, торгуя на основе общедоступной информации. С этого момента тесты ЕМН становятся совместными тестами рыночного поведения и моделей оценки стоимости капитала, так называемая совместная гипотеза (joint hypothesis). Таким образом, ЕМН сама по себе не может быть отвергнута. Помимо этого Ю. Фама выделил три формы рыночной эффективности (Fama 1970):

А) Слабая (weak) форма: информационное множество состоит только из информации, зафиксированной в прошлых ценах и объемах торгов.

Б) Умеренная (полусильная, средняя) (semi-strong) форма: информационное множество содержит всю общедоступную информацию (включая информацию о прошлых ценах и объемах торгов).

В) Сильная (strong) форма: цены отражают всю информацию, которая существует,

\footnotetext{
${ }^{30}$ Наблюдения Мандельброта положили начало попыткам применить математический инструментарий теории хаоса к анализу цен на финансовые активы. Согласно теории хаоса, большая часть явлений, представляющихся хаотичным набором случайностей, есть проявление скрытого от нас порядка и мельчайшие возмущения в нем часто оказываются причиной предустановленных крахов и устойчивого процветания рынков.
} 
включая инсайдерскую.

Суммируя академические исследования тестирования уровня информационной эффективности российского финансового рынка, отметим, что результаты весьма неоднозначны. В целом и на развитых рынках академических оппонентов ЕМН в избытке, на развивающихся же критика еще жестче.

С нашей точки зрения, российский рынок сегодня находится на пути к эффективности в средней форме, поскольку в условиях формирующейся институциональной среды и слабо развитой инфраструктуры финансового рынка очевидно, что цены на финансовые активы могут иметь систематические отклонения в какую-либо сторону от их внутренней стоимости. Что же касается слабой формы эффективности, то мы предельно скептичны к инструментарию технического анализа, что подтверждается множеством статистических исследований.

\section{Модели случайного блуждания}

Рассмотрим классификацию моделей случайного блуждания в терминах, предложенных Кэмпбеллом, Ло и Мак Кинли (Campbell, Lo and MacKinlay, 1996), относительно строгости предпосылок о распределении приращений $\xi_{\mathrm{t}+1}$.

- RW1 («Random walk 1»): $\xi_{\mathrm{t}+1}$ - IID (independently and identically distributed) распределены независимо и одинаково. Случайная величина $\mathrm{X}_{\mathrm{t}}$ блуждает случайно со смещением $\mu$, если:

где $\xi_{\mathrm{t}+1}-$ случайные

$$
X_{t+1}=X_{t}+\mu+\xi_{t+1}
$$

$\xi_{t+1}-$ случайные величины, которые распределены одинаково независимо, $E_{t} \xi_{t+1}=0, \operatorname{Var}_{t} \xi_{t+1}=\sigma^{2}, \operatorname{Cov}_{t}\left(\xi_{i} \xi_{j}\right)=0$, при $\mathrm{i} \neq \mathrm{j}$.

При $\mu=0$ (4) представляет собой мартингал, а $\Delta X_{t+1}$ - процесс справедливой игры. RW1 является более строгой с точки зрения недопущения предсказуемости, нежели модель справедливой игры, поскольку последняя не требует, чтобы статистические моменты выше первого были статистически независимы.

Чтобы избежать нарушения принципа ограниченной ответственности собственников (акция не может иметь отрицательную цену), при моделировании используют не сами цены $\left(\mathrm{P}_{\mathrm{t}}\right)$, а их натуральные логарифмы $\left(p_{t} \equiv \ln P_{t}\right)$ :

$$
p_{t+1}=p_{t}+\mu+\xi_{t+1}, \xi_{t+1} \sim I I D\left(0 ; \sigma^{2}\right) .
$$

При непрерывном временном ряде с доходностями ${ }^{31}$, распределенными нормально, независимо с нулевым средним и постоянной дисперсией $\sigma^{2}(5)$ является логнормальным вариантом модели Башелье.

- RW2: $\xi_{t+1}$ - INID (independent but not identically distributed) распределены независимо, но не одинаково:

$$
p_{t+1}=p_{t}+\mu+\xi_{t+1}, \xi_{t+1} \sim \operatorname{INID}\left(0 ; \sigma_{t}^{2}\right) .
$$

Таким образом, хотя RW2 слабее, чем RW1 (допускается наличие гетероскедастичности), оно по-прежнему сохраняет самые интересные экономические особенности RW1: любое будущее произвольное преобразование непрогнозируемо с помощью анализа любого произвольного преобразования прошлого приращения стоимости.

Зависимость от времени эмпирической дисперсии была впервые отмечена Кендаллом (Kendall, 1953) и впоследствии Мандельбротом (Mandelbrot, 1963). В то же время ряд

31 По определению, если мы используем логарифмы цен, то их разница будет эквивалентна доходности $\Delta p=p_{t+1}-p_{t}$ 
исследователей показывают, что предположение об одинаково распределенных приращениях цен на финансовые активы в долгосрочной перспективе не выглядит правдоподобным в силу бесчисленных изменений в экономической, технологической, институциональной среде (Schwert, 1989).

- RW3: $\xi_{\mathrm{t}+1}$ - NINID (not independent and not identically distributed) распределены не независимо и не одинаково. RW3 позволяет приращениям быть зависимыми, но некоррелированными и является самой слабой формой случайного блуждания. Простым примером процесса, который удовлетворяет условиям RW3, но не RW1 и RW2, является процесс, для которого $\operatorname{Cov}_{t}\left(\xi_{i} \xi_{j}\right)=0$, при $\mathrm{i} \neq \mathrm{j}$, но $\operatorname{Cov}_{t}\left(\xi^{2}{ }_{i}{ }^{2}{ }_{j}\right) \neq 0$, при $\mathrm{i} \neq \mathrm{j}$.

Дисперсия $\xi_{t+1}\left(\sigma^{2}{ }_{t+1}\right)$ может быть связана со своим предыдущим значением $\sigma_{t}^{2}$ (кластеризация волатильности), без нарушения свойств мартингальности. Получившие широкое распространение в 80-е годы модели динамики волатильности (ARCH Autoregressive Conditional Heteroskedasticity (Engle, 1982), GARCH Generalized Autoregressive Conditional Heteroskedasticity (Bollerslev, 1987) стали ответом на обнаружение кластеризации. Отметим, что данный эмпирический феномен не отвергает гипотезу рыночной эффективности. Ю. Фама писал: «Большие изменения цен следуют за большими изменениями, однако знак изменений непредсказуем. Это говорит о том, что важная информация не может быть оценена немедленно» (Fama, 1970).

Модель ARCH, предполагающая, что

$$
\sigma_{t}^{2}=\alpha_{0}+\alpha_{1} \xi_{t-1}^{2}+\ldots+\alpha_{n} \xi_{t-n}^{2}
$$

дала возможность идентифицировать эффект кластеризации. Однако модель не дает объяснения того, в какую сторону будут двигаться цены, то есть не предоставляет информации о знаке $\xi_{t}$. Отметим, что ARCH-модель породила огромное количество сходных моделей, направленных на то, чтобы «ухватить» эффект кластеризации.

\section{«Сбои» рыночной эффективности (рыночные аномалии)}

Все возрастающее количество исследований ЕMН привело к статистическому обнаружению примеров рыночного поведения, которые несовместимы с существующими моделями риска и доходности. Мы провели обзор эмпирических работ, исследующих наиболее часто наблюдаемые аномалии и дававшиеся им объяснения, однако, в силу ограничений на объем статьи, вынуждены его опустить. Впрочем, выявлено огромное множество «как будто» рыночных аномалий, статистическое обнаружение которых обусловлено грубейшими методологическими нарушениями (зачастую инвестиционные стратегии, выстраиваемые исследователями в целях тестирования ЕМН, игнорируют операционные издержки, корректировки на риск, дивидендные выплаты, непредвзятость в составлении выборки и др.).

Отметим, что сегодня наиболее мощная критика исходит от финансовых бихевиористов, которые утверждают, что ЕМН зиждется на совершенно неверной предпосылке относительно человеческого поведения, его рациональности. Тем не менее, вопреки распространенному мнению, ЕМН не требует, чтобы все участники рынка были рациональны. На самом деле рынки могут быть эффективными даже тогда, когда некоторые группы инвесторов являются иррациональными и взаимосвязанными. ЕМН выдерживается до тех пор, пока есть некоторые рациональные инвесторы ${ }^{32}$ и возможности совершения арбитражных операций (Shleifer, 2000).

В то же время, когда рыночные аномалии становятся общеизвестными, инвесторы

\footnotetext{
${ }^{32}$ К ним следует отнести в первую очередь тысячи высокопрофессиональных аналитиков, постоянно держащих руку на пульсе рынка и готовых посоревноваться в умении предсказывать и проницательности с менее искушенными инвесторами.
} 
начинают использовать основанные на них стратегии, тем самым ослабляя и устраняя аномалии (Schwert, 2002). Иными словами, постоянный поиск неэффективностей делает рынок эффективнее. Либо же ослабление «аномалий» обусловлено их внутренней мифичностью, поскольку в распоряжении исследователей имеется большой массив финансовых данных и нормальная тенденция, что они сосредоточивают свое внимание на поиске сбоев ЕMH, и время от времени будут называться отдельные проблемы, дающие статистически значимые результаты. Тонкое замечание сделал Бернстайн: «Возможно, существуют инвесторы, которые постоянно переигрывают рынок, и не в силу удачи, а благодаря пониманию, но они упорно держатся в непроницаемой тени». Но в это трудно поверить.

\section{Создание стоимости в условиях низкоэффективных рынков}

Современные технологии управления на основе критерия стоимости зиждятся на допущениях достаточно эффективных рынков капитала. Как же быть менеджеру при нарушении тождества рыночной цены активов и их внутренней стоимости? Т. Коупленд, Т. Коллер, Дж. Муррин считают: «Если рынок даже и проявляет временами неэффективность, менеджерам в принятии решений следует исходить из предпосылки, что рынок эффективен» (Коупленд, 2005). Они конечно же рассуждали с позиции развитого рынка и исходили из того, что рынок в долгосрочной перспективе устранит дисбалансы. В условиях же развивающегося рынка, в долгосрочной перспективе мы все скорее умрем, как иронизировал Дж.М. Кейнс, поэтому могут возникать финансовые рычаги создания стоимости.

Имеется значительный пласт эмпирических исследований, показывающих иррелевантность на эффективных финансовых рынках ряда корпоративных действий. Эффективные рынки не вознаграждают компании за стабильность и предсказуемость прибылей, поэтому менеджеры не должны пытаться сгладить прибыль во времени или «подгонять» прибыль к целевым уровням аналитиков, которые часто устанавливаются произвольно (Koller and Rajan, 2002; Graham et al., 2005).

Для эффективного рынка иррелевантны бухгалтерские способы отражения фундаментальных показателей компании, поэтому менеджерам не нужно беспокоиться об отражении в учете расходов по опционным программам или о новых правилах бухгалтерского учета (Lindenberg and Ross, 1999; Aboody et al., 2004).

Также инвесторы заинтересованы в «содержании», а не в «форме» их акций. На развитых рынках менеджерам не стоит ожидать прироста стоимости от дробления акций, листинга на других рынках, выпуска смешанных форм ценных бумаг (например, конвертируемых облигаций) (Brennan and Copeland, 1988; Boehme and Danielsen, 2007; Dobbs and Goedhart, 2008).

Впрочем, высокая эффективность финансовых рынков не означает, что финансовый менеджер должен пустить финансирование на самотек; в то же время ключевые рычаги наращивания стоимости находятся в левой стороне баланса.

Можно выделить несколько очевидных следствий неэффективного функционирования рынков, влияющих на решения менеджмента:

1. Важен фактор времени эмиссии акций. На эффективном рынке цена акций равна их внутренней стоимости, следовательно, при дополнительной эмиссии прежние акционеры ничего не теряют, так как предприятие получает ровно столько, сколько стоимости дополнительно распределяется (опуская трансакционные затраты) ${ }^{33}$. Однако если рыночная эффективность нарушается, то позиция менеджмента в зависимости от отклонения рыночной цены от внутренней стоимости очевидна: при переоцененности фирмы предприимчивые менеджеры будут заинтересованы в немедленном выпуске акций (либо в употреблении части

\footnotetext{
33 Также, впрочем, как ничего и не выигрывают. В данном случае отношения прежних и новых акционеров справедливо представить в виде игры «с нулевой суммой», то есть выигрыш одной стороны возможен лишь за счет противоположной.
} 
акций для оплаты поглощения), при недооценке - предпочтут повременить (или же выкупить часть собственных акций $)^{34}$.

2. На эффективных рынках инвесторы заинтересованы в «содержании», а не в «форме» их акций, если рынок работает со сбоями в эффективности, менеджмент может провернуть выгодную сделку по финансированию за счет изменения «формы» эмитируемых ценных бумаг.

3. Финансовые и инвестиционные решения не следует рассматривать порознь. Искажения действительной стоимости фирмы неэффективными рынками могут привести к выбраковыванию привлекательных при эффективной работе рынка инвестиционных проектов.

4. Искажены результаты использования инструментов оценки требуемой инвесторами доходности (модели САРМ, арбитражной модели и др.), исходящих из предпосылки эффективности рынка. Также дополнительные ограничения возникают при применении широко используемой в инвестиционном анализе для оценки реальных опционов модели Блэка - Шоулза.

5. В качестве дополнительного истинного довода при поглощении другой компании может быть глубокая уверенность менеджмента в недооцененности акций «жертвы».

\section{Заключение}

На совершенно конкурентном рынке каждый продавец получает нормальную прибыль, то есть прибыль, достаточную для покрытия затрат на капитал, но недостаточную для появления новых конкурентов. Если спроецировать данные доводы на рынок капитала, то следует, что любая новая информация мгновенно находит отражение в цене. Иными словами, ожидаемая доходность активов будет равна альтернативным издержкам инвестирования в данные активы (рыночная цена есть непредвзятая оценка фундаментальной стоимости).

Эффективность финансовых рынков не означает, что финансовый менеджер не должен уделять внимания финансовым решениям; в то же время создание стоимости - это продукт состоятельных инвестиционных решений (поиск неэффективностей на реальных рынках), генерирующих экономические ренты.

Результаты тестирования отечественного рынка капитала достаточно противоречивы, тем не менее, судя по всему, российский рынок в условиях формирующейся институциональной среды и слаборазвитой инфраструктуры финансового рынка эффективен в слабой, но не в средней форме. На таких рынках появляются резервы наращивания стоимости за счет финансовых рычагов, которыми обделены компании в условиях высокоэффективных финансовых рынков развитых стран.

\section{Список литературы}

1. Брейли Р., Майерс С. Принципы корпоративных финансов / Пер. с англ. Н. Барышниковой. - М: ЗАО «Олимп-Бизнес», 2008.

2. Коупленд Т., Коллер Т, Муррин Дж. Стоимость компаний: оценка и управление. - 3-е изд., перераб. и доп. - М.: ЗАО «Олим-Бизнес», 2005.

3. Фабоций Ф. Управление инвестициями: Пер. с англ. - М.: ИНФРА-М, 2000.

4. Ширяев А.Н.Основы стохастической финансовой математики. Том I: Факты. Модели / А.Н. Ширяев. - М.: ФАЗИС, 1998.

5. Aboody, B., Barth, M., and Kasznik, R. (2004), Firms' Voluntary Recognition of StockBased Compensation Expense, Journal of Accounting Research, 42 (2004) 251-275.

\footnotetext{
34 Информационный негативный сигнал дополнительного выпуска акций характерен и для конкурентных западных рынков, что объясняется отсутствием веры инвесторов в сильную форму эффективности рынков и их подозрениями в отношении менеджмента, обладающего большей информацией.
} 
6. Akgiray, V., and Booth, G. (1988), The stable-law model of stock returns, Journal of Business and Economic Statistics, 6 (1988) 51-57.

7. Alexander, S. (1961), Price Movements in Speculative Markets: Trends or Random Walks, Industrial Management Review, 2(2) (1961) 7-26.

8. Alexander, S. (1964), Price Movements in Speculative Markets: Trends or Random Walks, No. 2. Industrial Management Review, 5(2) (1964) 25-46.

9. Andreou, E., and Pittis, N., and Spanos, A. (2001), On Modelling Speculative Prices: The Empirical Literature, Journal of Economic Surveys, 15(2) (2001) 187-220.

10. Bachelier, L. (1900), Théorie de la Spéculation (English Translation; Cootner (ed.), 1964. Random Character of Stock Market Prices, Massachusetts Institute of Technology, 17-78).

11. Blattberg, R., and Gonedes, N. (1974), A comparison of Stable and Student's t distributions as statistical models for stock prices, Journal of Business, 47 (1974) 244-280.

12. Boehme, R., and Danielsen, B.R. (2007), Stock-Split Post-Announcement Returns: Underreaction or Market Friction? Financial Review, 42 (2007) 485-506.

13. Bollerslev, T. (1987), A conditionally heteroskedastic time series model for speculative prices and rates of return, Review of Economics and Statistics, 69 (1987) 542-547.

14. Bollerslev, T., Chou, R., and Kroner, K. (1992), ARCH modeling in finance: A review of the theory and empirical evidence, Journal of Econometrics, 52 (1992) 5-59.

15. Brennan, M., and Copeland, T. (1988), Stock Splits, Stock Prices, and Transaction Costs, Journal of Financial Economics, 22 (1988) 83-101.

16. Campbell, J., Lo, A., and MacKinlay, C. (1997), The Econometrics of Financial Markets. Princeton University Press.

17. Cowles, A. (1933), Can Stock Market Forecasters Forecast? Econometrica, 1(3) (1933) 309-324.

18. Cowles, A., and Jones, H. (1937), Some A Posteriori Probabilities in Stock Market Action, Econometrica, 5(3) (1937) 280-294.

19. Dobbs, R., and Koller, T. (2006), Inside a Hedge Fund: An Interview with the Managing Partner of MaverickCapital, McKinsey on Finance, 19 (2006) 6-11.

20. Engle, R. (1982), Autoregressive conditional heteroskedasticity with estimates of the variance of United Kingdom inflation, Econometrica, 50 (1982) 987-1008.

21. Fama, E. (1965), Random Walks in Stock Market Prices, Financial Analysts Journal, 21(5) (1965) 55-59.

22. Fama, E. (1970), Efficient Capital Markets: A Review of Theory and Empirical Work, Journal of Finance, 25(2) (1970) 383-417.

23. Fama, E. (1991), Efficient Capital Markets: II, Journal of Finance, 46(5) (1991) 1575-1617.

24. Graham, J., Harvey, C., Rajgopal, S. (2005), The Economic Implications of Corporate Financial Reporting, Journal of Accounting and Economics, 40 (2005) 3-73.

25. Granger, C., and Morgenstern, O. (1963), Spectral Analysis of New York Stock Market Prices, Kyklos, 16 (1963) 1-27.

26. Hagerman, R., and Richmond, R. (1973), Random Walks, Martingales and the OTC, Journal of Finance, 28(4) (1973) 897-909.

27. Jensen, M. (1978), Some anomalous evidence regarding market efficiency, Journal of Financial Economics, 6(2-3) (1978) 95-101.

28. Kendall, M. G. (1953), The Analysis of Economic Time-Series - Part I: Prices, Journal of the Royal Statistical Society. Series A (General), 116(1) (1953) 11-25.

29. Koller, T., and Rajan, S. (2002), Who's Afraid of Variable Earnings? McKinsey on Finance, 4 (2002) 13-17.

30. Koller, T., Goedhart, M., Wessels, D. (2010), Valuation: Measuring and Managing the Value of Companies, 5th Edition (Wiley Finance).

31. Lindenberg, E., and Ross, M. (1999), To Purchase or to Pool: Does It Matter? Journal of Applied Corporate Finance, 12 (1999) 32-47.

32. MacCauley, F. (1925), Forecasting Security Prices, Journal of the American Statistical 
Association, 20(150) (1925) 244-249.

33. Mandelbrot, B. (1963), The Variation of Certain Speculative Prices, The Journal of Business, 36(4) (1963) 394-419.

34. Mandelbrot, B. (1966), Forecasts of Future Prices, Unbiased Markets, and "Martingale" Models, Journal of Business, 39(1) (1966) 242-255.

35. Mandelbrot, B. (1969), Long-run linearity, locally Gaussian process, H-spectra and infinite variances, International Economic Review, 10 (1969) 82-111.

36. Mandelbrot, B. (1997), Fractals and Scaling in Finance: Discontinuity, Concentration, Risk. Springer.

37. Merton, R. (1990) Continuous-Time Finance. Basil Blackwell.

38. Moore, A. (1962), A Statistical Analysis of Common Stock Prices, unpublished Ph. D. Dissertation, Graduate School of Business, University of Chicago.

39. Osbourne, M. (1959), Brownian Motion in the Stock Market, Operations Research, 7(2) (1959) 145-173.

40. Pagan, A. R. (1996), The econometrics of financial markets, Journal of Empirical Finance, 3 (1996) 15-102.

41. Pearson, K. (1905), The Problem of the Random Walk, Nature, 72(1865) (1905) 294-324.

42. Roberts, H. (1959), Stock-Market "Patterns" and Financial Analysis: Methodological Suggestions, Journal of Finance, 14(1) (1959) 1-10.

43. Samuelson, P. (1965), Proof that properly anticipated prices fluctuate randomly, Industrial Management Review, 6 (1965) 41-49.

44. Samuelson, P. (1973), Mathematics of Speculative Price, SIAM Review, 15(1) (1973) 1-42.

45. Sawyer, K. R. (1993), Continuous time financial models: statistical applications of stochastic processes. Handbook of statistics, Vol. 0.11 .

46. Schwert, W. (1989), Why does stock market volatility change over time? Journal of Finance, 44 (1989) 1115-1153.

47. Schwert, W. (2002), Anomalies and Market Efficiency. Handbook of the Economics of Finance.

48. Shleifer, A. (2000), Inefficient Markets, An Introduction to Behavioral Finance. Published in the United States by Oxford University Press Inc.

49. Tucker, A. (1992), A reexamination of finite and infinite-variance distributions as models of daily stock returns, Journal of Business and Economic Statistics, 10 (1992) 73-81.

50. Working, H. (1934), A Random-Difference Series for Use in the Analysis of Time Series, Journal of the American Statistical Association, 29(185) (1934) 11-24.

51. Working, H. (1949), The Investigation of Economic Expectations, The American Economic Review, 39(3) (1949) 150-166.

52. Working, H. (1960), Note on the Correlation of First Differences of Averages in a Random Chain, Econometrica, 28(4) (1960) 916-918. 